\title{
Establishment of ultrasound as a diagnostic aid in the referral of patients with abdominal pain in an emergency department - a pilot study
}

This article was published in the following Dove Press journal:

Open Access Emergency Medicine

25 March 2015

Number of times this article has been viewed

\section{Liv la Cour Poulsen'}

Emilie Stokholm Bækgaard'

Per Grosen Istre'

Thomas Andersen Schmidt ${ }^{\prime}$

Torben Larsen ${ }^{2}$

'Emergency Department, Copenhagen University Hospital, Holbaek, Denmark; ${ }^{2}$ Department of Obstetrics and Gynecology, Copenhagen University Hospital, Holbaek, Denmark
Correspondence: Liv la Cour Poulsen Emergency Department, Copenhagen University Hospital, Smedelundsgade 60, 4300 Holbæk, Denmark

Tel+45616000 4l

Email livlacour@hotmail.com
Purpose: Ultrasonography is a noninvasive, cheap, and fast way of assessing abdominal pain in an emergency department. Many physicians working in emergency departments do not have pre-existing ultrasound experience. The purpose of this study was to investigate the ability of first-year internship doctors to perform a reliable ultrasound examination on patients with abdominal pain in an emergency setting.

Materials and methods: This study took place in an emergency department in Denmark. Following a 1-day ultrasound introduction course, three doctors without prior ultrasound experience scanned 45 patients during a 2-month period. The applicability of the examinations was evaluated by subsequent control examination: computed tomography, operation, or ultrasound by a trained radiologist or gynecologist or, in cases where the patient was immediately discharged, by ultrasound image evaluation.

Results: In 14 out of 21 patients with a control examination, there was diagnostic agreement between the project ultrasound examination and the control. Image evaluation of all patients showed useful images of the gallbladder, kidneys, liver, abdominal aorta, and urinary bladder, but no useful images for either the pancreas or colon.

Conclusion: With only little formal training, it is possible for first-year internship doctors to correctly visualize some abdominal organs with ultrasonography. However, a longer study time frame, including more patients, and an ultrasound course specifically designed for the purpose of use in an emergency department, is needed to enhance the results.

Keywords: ultrasound training, emergency medicine, diagnostic imaging, acute abdomen

\section{Introduction}

Acute abdominal pain is one of the more common presentations in the emergency department (ED). ${ }^{1}$ Early diagnosis is important. The origin of pain may be surgical or medical, and it may range from benign, self-limiting conditions, to diseases of high mortality. Correct diagnosis and treatment are therefore important, ${ }^{2}$ as is further referral of the patient to the correct department for subsequent treatment (or, indeed, to discharge the patient). Patient history, physical examination, and laboratory tests are the primary steps, but they are not always reliable or specific enough to pinpoint an exact diagnosis.

Computed tomography (CT) and/or magnetic resonance imaging (MRI) scans are widely considered as the gold standard for diagnostic accuracy, but both have their drawbacks, including the administration of a considerable radiation dose to the patient in the case of CT scan, and potential for renal complications from the use of MRI contrast, such as gadolinium. ${ }^{3}$ Ultrasonography (US), on the other hand, is noninvasive, 
cheap, requires neither the injection of a contrast medium nor radiation, and can be employed quickly as a bedside examination tool. ${ }^{4}$ One study has shown that patients assessed with US at ED have a shorter length of stay in the department. ${ }^{5}$ These properties make US a desirable initial choice of imaging modality in the attempt to reach the correct diagnosis in an emergency setting.

US already has a well-established role in the diagnostic assessment of the acute abdomen; ${ }^{4}$ however, in most hospitals, the procedure is performed by sonographers, radiologists, or other physicians with broad US experience who do not necessarily function as ED staff, and they may have limited or no on-call duties. It has, however, been suggested that even physicians without pre-existing US experience can perform valid and reliable examinations of, for example, the gallbladder. ${ }^{6}$ Earlier studies have indicated an increase in diagnostic sensitivity and specificity among younger physicians after completing short ultrasound training courses of, for example, 4-16 hours..$^{7-9}$ Furthermore, recent studies have showed promising results when evaluating the ability of residents and medical students to perform US examinations with a pocket ultrasound device after a short training period. ${ }^{10-12}$

In the United States, the need for training in emergency US is recognized, starting with undergraduate medical education through postgraduate training, and into residency programs for a thorough integration of US practice in EDs of all sizes. ${ }^{13}$ In Denmark, US does not yet hold an established role in EDs. ${ }^{14}$ Younger physicians, often first-year internship doctors, mostly staff the departments across the country and, hence, comprehensive implementation of US at the EDs should purportedly include these physicians.

The purpose of the current study is to highlight the impact of US performed by younger physicians in the ED in an attempt to evaluate its future role in EDs.

\section{Materials and methods}

\section{Study design}

Three first-year postgraduate physicians attended a 1-day introduction course on practical US. Their ability to perform bedside abdominal and vaginal US in an emergency setting over a 2-month period was prospectively evaluated.

\section{Setting and population}

The study took place in the ED at the Copenhagen University Hospital situated in Holbaek, Denmark, during December 2013 and January 2014. This department has an important role in the education of first-year postgraduate physicians who work in the department for a 6-month period as part of their internship. All admissions to the hospital come through the ED for evaluation, diagnostics, initial treatment, and further transfer to specialized departments. A small percentage of patients are directly discharged from this unit.

We included all patients who were admitted with abdominal pain, and who were triaged "green" or "yellow", indicating that they were hemodynamically stable and in a condition that allowed a bedside US to be performed by one of the project physicians without compromising patient safety. Only patients above 18 years were included, and for vaginal scans, only nonvirgin females with lower abdominal pain were included.

\section{Ultrasound training course}

The course was arranged by the Danish Ultrasound Diagnostic Society and was primarily focused on the use of vaginal US, with a shorter part of the day focused on abdominal US. The course consisted of an 8-hour introduction to the use of US, including lessons on the technicalities of US equipment, image optimization, and anatomy and physics of both the abdomen and pelvis. Secondly, practical sessions were undertaken, during which participants were taught to perform an US examination in a practical laboratory setting in 4-5-person groups. Each participant practiced a particular examination while the others observed. Healthy volunteers were hired to be the models (be examined).

\section{Study protocol}

The protocol consisted of three elements. When a patient was admitted to the ED with abdominal pain, a physician in the ED initially examined him or her. The physician, who was not necessarily a project physician, provided the patient with a tentative diagnosis based on the symptoms, clinical examination, and blood test results. Thereafter, one of the project physicians performed a bedside ultrasound examination (project US examination) with the purpose of visualizing the anatomical structures and identifying possible abnormalities, as listed in Table 1. The results were marked on an inclusion form by tick-off. Informed consent was obtained from all the patients included in the study.

Findings on ultrasound examination were blinded to both the patient and the treating physician, and therefore had no influence on the following admission and treatment. Images, or short-film sequences of positive findings, were saved for later evaluation.

Thirdly, the medical records of the included patients were reviewed to identify those patients who underwent one of three subsequent additional diagnostic examinations or procedures: surgery; CT/MRI scan; or formal ultrasound by a trained gynecologist or radiologist. A true-positive and true-negative result was defined as agreement between the 
Table I Ultrasound focus

\begin{tabular}{ll}
\hline Organ & Findings \\
\hline Gallbladder, AB & Normal, thickening of wall, stones \\
Liver, AB & Normal, tumor, abscess, cysts \\
Pancreas, AB & Normal, inflammation, cysts \\
Abdominal aorta, AB & Normal, aneurysm \\
Kidneys, AB & Normal, hydronephrosis, cysts \\
Appendix, AB & Normal, thickening of wall $(>3 \mathrm{~mm})$, abscess \\
Colon diverticula, AB & Normal, thickening of wall $(>3 \mathrm{~mm})$, abscess \\
Bladder, AB/VG & Normal, urinary retention, stones, tumor \\
Ovaries, VG & Normal, cysts $>25$ mm \\
Uterus, VG & Normal, fibromas, thickening of the \\
Small pelvis, VG & endometrium, intrauterine pregnancy \\
\hline
\end{tabular}

Abbreviations: $A B$, abdominal ultrasound; $V G$, vaginal ultrasound.

ultrasound findings and the findings obtained from one of these three "gold-standards".

One project author, TL, evaluated the ultrasound pictures and short-film sequences retrospectively. Images from patients who were discharged without further examination were also included in this evaluation.

\section{Results}

Over the 2-month period, 45 patients were included, of which seven patients could not participate due to dementia (three patients), intoxication (two patients), severe pain during ultrasound (one patient), or allergic reaction to the ultrasound gel (one patient). The project physicians scanned 38 patients.
Twenty-one subsequently underwent operation, CT, or formal US by a trained gynecologist or radiologist, and the remaining 17 patients were discharged without further examination. Images or short-film sequences from the project ultrasound were evaluated for all 38 patients.

Agreement between the project ultrasound and control examination were found in 14 of the 21 patients, where the diagnosis was cholecystolithiasis, nephrolithiasis, appendicitis, abdominal aortic aneurysm, or "no positive finding" (Table 2). Lack of agreement was found in seven patients, all of these due to an evaluation of "no positive finding" on the project ultrasound, where a positive finding was found on CT of most frequent kidney stones (four out of seven cases). Subsequent image evaluation of all the included patients concluded that there was consistently useful US imaging of the gallbladder and kidneys, both normal and diseased, and many acceptable images of the liver, abdominal aorta, and urinary bladder. No useful images were present for either the pancreas or colon, and too few patients had a vaginal scan to conclude any effective vaginal ultrasound skills.

\section{Discussion}

This pilot study was intended to put focus on the role and use of US in the hands of younger physicians in an ED. The time frame and subsequent data that were obtained proved too narrow to conclude any effect of the use of US in the hands of these first-year doctors. However, both the evaluation of the

Table 2 Project ultrasound versus control

\begin{tabular}{|c|c|c|c|c|}
\hline Patient ID & Project US & CT & Control ultrasound & Operation \\
\hline 4 & Nephrolithiasis & Nephrolithiasis & - & - \\
\hline 5 & Thickening of appendix & - & - & Appendicitis \\
\hline 8 & No positive finding & Nephrolithiasis & - & - \\
\hline 9 & No positive finding & Nephrolithiasis & & - \\
\hline II & Cholecystolithiasis & - & - & Cholecystolithiasis \\
\hline 13 & $\begin{array}{l}\text { Cholecystolithiasis and wall } \\
\text { thickening }\end{array}$ & - & - & $\begin{array}{l}\text { Cholecystolithiasis and } \\
\text { cholecystitis }\end{array}$ \\
\hline 15 & No positive finding & - & No positive finding & - \\
\hline 17 & No positive finding & - & No positive finding & - \\
\hline 18 & Periappendiceal abscess & - & - & Periappendiceal abscess \\
\hline 19 & No positive finding & - & Cholecystolithiasis & - \\
\hline 21 & $\begin{array}{l}\text { AAA } 8 \times 7 \mathrm{~cm} \text { and two cysts, } \\
\text { right kidney }\end{array}$ & $\begin{array}{l}\text { AAA } 7.7 \mathrm{~cm} \text { and } \\
\text { cysts, right kidney }\end{array}$ & - & - \\
\hline 27 & Free fluid in Pouch of Douglas & - & Free fluid in Pouch of Douglas & Normal appendix \\
\hline 31 & No positive finding & Nephrolithiasis & - & - \\
\hline 32 & No positive finding & - & No positive finding & - \\
\hline 34 & No positive finding & lleus & - & - \\
\hline 35 & Cholecystolithiasis & - & Cholecystolithiasis & Cholecystolithiasis \\
\hline 38 & No positive finding & - & No positive finding & - \\
\hline 40 & No positive finding & No positive finding & - & - \\
\hline 43 & No positive finding & - & - & Appendicitis \\
\hline 44 & No positive finding & Nephrolithiasis & - & - \\
\hline 45 & No positive finding & & & No positive finding \\
\hline
\end{tabular}

Abbreviations: ID, identifier; US, ultrasonography; CT, computed tomography; AAA, abdominal aortic aneurysm. 
images by a specialist and the comparison of these images to "gold standard" examinations showed that with little formal training, young physicians might be able to use US in a clinical setting. Not surprisingly, it was found that some organs are more difficult to visualize after limited training than others, and an important drawback of this project included the fact that the US course that these physicians attended was not specifically targeted at this project or, indeed, at the use of abdominal US. A more extended course, focusing on the specific needs in an ED setting and focusing on fewer organs, is most likely required to improve US skills and enhance results in this population of physicians.

In other countries, such as the United Kingdom and the United States, US is described as being used consistently and increasingly in the EDs, at the bedside, and by all physicians and medical students; US may also include small, handheld devices. Some go as far as to argue that US should be considered "the new stethoscope", ${ }^{15}$

The Danish Society for Emergency Medicine (DASEM) recently published its first recommendation for the use of clinical ultrasound in EDs, ${ }^{14}$ pinpointing which US procedures should be second nature to the ED physician, and how the physician should accomplish the associated skills and become certified. However, these guidelines are oriented at physicians with ED specialty training and tenure, forgetting that it is primarily first-year postgraduate physicians that work in EDs.

The combination of senior physicians with varying levels of US skill, as well as a consistent first-line evaluation of patients by first-year postgraduate physicians with no formal US training, makes it difficult, if not impossible, to implement consistent use of bedside US in the diagnosis and referral of patients arriving at the ED. It will be difficult to establish US in the EDs thoroughly without including the younger doctors in formal US training.

To move forward in the process of bringing US closer to being an everyday diagnostic tool in an ED setting, we have to discuss the learning possibilities and options for providing US training to younger doctors, as well as to discuss the general willingness to incorporate bedside US in EDs. Furthermore, there is a need for more thorough investigation as to which medical and/or surgical conditions point-of-care US in the ED can and should be used to diagnose, or exclude, with a minimum of errors.

\section{Conclusion}

Our study showed that with little formal training, it is possible for first-year internship doctors to correctly visualize some abdominal organs with US, but that a longer timeframe, the inclusion of more patients, and a course specifically designed for the purpose of the use of ultrasound in an ED, are needed to enhance the results.

Continuous formal training of all physicians working in the ED, the evaluation of subsequent point-of-care US, and ongoing adjustment and re-evaluation of training, will possibly bring the EDs closer to optimally exploiting the possibilities of ultrasound in the evaluation of patients with abdominal pain.

\section{Author contributions}

All authors contributed toward data analysis, designing, drafting, and critically revising the paper, gave final approval of the version to be published, and agree to be accountable for all aspects of the work.

\section{Disclosure}

The authors report no conflicts of interest in this work.

\section{References}

1. Powers RD, Guertler AT. Abdominal pain in the ED: stability and change over 20 years. Am J Emerg Med. 1995;13(3):301-303.

2. Rosen MP, Sands DZ, Longmaid HE 3rd, Reynolds KF, Wagner M, Raptopoulos V. Impact of abdominal CT on the management of patients presenting to the emergency department with acute abdominal pain. AJR Am J Roentgenol. 2000;174(5):1391-1396.

3. Stoker J, van Randen A, Lameris W, Boermeester MA. Imaging patients with acute abdominal pain. Radiology. 2009;253(1):31-46.

4. Puylaert JB. Ultrasonography of the acute abdomen: gastrointestinal conditions. Radiol Clin North Am. 2003;41(6):1227-1242, vii.

5. Blaivas M, Harwood RA, Lambert MJ. Decreasing length of stay with emergency ultrasound examination of the gallbladder. Acad Emerg Med. 1999;6(10):1020-1023.

6. Eiberg JP, Grantcharov TP, Eriksen JR, et al. Ultrasound of the acute abdomen performed by surgeons in training. Minerva Chir. 2008;63(1): $17-22$.

7. Lanoix R, Baker WE, Mele JM, Dharmarajan L. Evaluation of an instructional model for emergency ultrasonography. Acad Emerg Med. 1998;5(1):58-63.

8. Mandavia DP, Aragona J, Chan L, Chan D, Henderson SO. Ultrasound training for emergency physicians - a prospective study. Acad Emerg Med. 2000;7(9):1008-1014.

9. Torres-Macho J, Antón-Santos JM, García-Gutierrez I, et al; Working Group of Clinical Ultrasound, Spanish Society of Internal Medicine. Initial accuracy of bedside ultrasound performed by emergency physicians for multiple indications after a short training period. Am J Emerg Med. 2012;30(9):1943-1949.

10. Andersen GN, Viset A, Mjølstad OC, Salvesen O, Dalen H, Haugen BO. Feasibility and accuracy of point-of-care pocket-size ultrasonography performed by medical students. BMC Med Educ. 2014;14:156.

11. Carrié C, Biais M, Lafitte S, Grenier N, Revel P, Janvier G. Goal-directed ultrasound in emergency medicine: evaluation of a specific training program using an ultrasonic stethoscope. Eur J Emerg Med. Epub June 6, 2014.

12. Mjølstad OC, Andersen GN, Dalen H, et al. Feasibility and reliability of point-of-care pocket-size echocardiography performed by medical residents. Eur Heart J Cardiovasc Imaging. 2013;14(12): 1195-1202. 
13. American College of Emergency Physicians. Emergency ultrasound guidelines. Ann Emerg Med. 2009;53(4):550-570.

14. Laursen CB, Nielsen K, Riishede M, et al. A framework for implementation, education, research and clinical use of ultrasound in emergency departments by the Danish Society for Emergency Medicine. Scand J Trauma Resusc Emerg Med. 2014;22:25.
15. Solomon SD, Saldana F. Point-of-care ultrasound in medical educationstop listening and look. N Engl J Med. 2014;370(12):1083-1085.

Open Access Emergency Medicine

\section{Publish your work in this journal}

Open Access Emergency Medicine is an international, peer-reviewed, open access journal publishing original research, reports, editorials, reviews and commentaries on all aspects of emergency medicine. The manuscript management system is completely online and includes a very quick and fair peer-review system, which is all easy to use.
Dovepress

Visit http://www.dovepress.com/testimonials.php to read real quotes from published authors. 